OPEN ACCESS

Edited by:

Xiaoqiang Tang,

Sichuan University, China

Reviewed by:

Lanlan Zhang,

Sichuan University, China

Claudia Penna,

University of Turin, Italy

Meera G. Nair,

University of California, Riverside,

United States

*Correspondence:

Wenjuan Liu

Iwj0320@szu.edu.cn

Specialty section:

This article was submitted to

Cellular Biochemistry,

a section of the journal

Frontiers in Cell and Developmental

Biology

Received: 07 April 2021

Accepted: 23 June 2021

Published: 15 July 2021

Citation:

Lv M and Liu W (2021)

Hypoxia-Induced Mitogenic Factor: A Multifunctional Protein Involved

in Health and Disease.

Front. Cell Dev. Biol. 9:691774. doi: 10.3389/fcell.2021.691774

\section{Hypoxia-Induced Mitogenic Factor: A Multifunctional Protein Involved in Health and Disease}

\author{
Moyang $L v^{1}$ and Wenjuan $L^{2} u^{2 *}$ \\ ${ }^{1}$ Department of Gastroenterology, Xinqiao Hospital, Third Military Medical University, Chongqing, China, ${ }^{2}$ Department \\ of Pathophysiology, Health Science Center, Shenzhen University, Shenzhen, China
}

Hypoxia-induced mitogenic factor (HIMF), also known as resistin-like molecule $\alpha$ $(R E L M \alpha)$ or found in inflammatory zone 1 (FIZZ1) is a member of the RELM protein family expressed in mice. It is involved in a plethora of physiological processes, including mitogenesis, angiogenesis, inflammation, and vasoconstriction. HIMF expression can be stimulated under pathological conditions and this plays a critical role in pulmonary, cardiovascular and metabolic disorders. The present review summarizes the molecular characteristics, and the physiological and pathological roles of HIMF in normal and diseased conditions. The potential clinical significance of these findings for human is also discussed.

Keywords: hypoxia-induced mitogenic factor, mitogenesis, angiogenesis, proinflammation, vasoconstriction

\section{INTRODUCTION}

Hypoxia-induced mitogenic factor (HIMF), also known as found in inflammatory zone 1 (FIZZ1) or resistin-like molecule $\alpha($ RELM $\alpha)$, is a pro-inflammatory cytokine in mice. HIMF expression is markedly increased in the hyperplastic bronchial epithelium of mice with allergic pulmonary inflammation (Holcomb et al., 2000). Three years later, Teng et al. (2003) also reported a novel role for this molecule in the pathogenesis of hypoxia-induced pulmonary hypertension ( $\mathrm{PH})$. Hypoxia was found to induce HIMF expression, and the protein was upregulated in a murine chronic hypoxia model of pulmonary hypertension. The authors renamed this gene HIMF, as the recombinant protein stimulated the proliferation of rat pulmonary microvascular smooth muscle cells (PSMCs). Following investigations using a chronic hypoxia-induced PH mouse model uncovered a pluoripotent array of pathological roles played by HIMF, including in angiogenesis, vasoconstriction, inflammation and fibrosis. Together, these properties endow HIMF with a critical function in pulmonary vascular remodeling and, thus, in the development of $\mathrm{PH}$ after hypoxia. Notably, HIMF has also been linked to both cell survival and cell death, depending on the dose and cellular context. For example, despite the harmful effects of increased HIMF expression in lung disease, particularly in $\mathrm{PH}$, basal expression of HIMF is required for normal lung development.

In addition to its involvement in pulmonary disease, a number of studies have demonstrated that HIMF also participates in the development of cardiovascular diseases and metabolic disorders in mice. Furthermore, recent studies by our group have uncovered a novel role for HIMF in the pathogenesis of pressure overload-induced cardiac hypertrophy and fibrosis (Kumar et al., 2018, 2019). These findings are of particular note because the two human homologs of HIMF, $\operatorname{RELM} \beta$, and resistin (hRETN), are also associated with cardiovascular diseases. For example, there is a positive correlation between resistin plasma levels and the incidence of cardiovascular 
events in patients with cardiovascular diseases (Zhang et al., 2011). Furthermore, studies in mice have revealed a causal relationship between the RELM $\beta$ expression and the pathogenesis of atherosclerosis (Kushiyama et al., 2013). Therefore, HIMF (and, by extension, its human homologs) may represent potential, clinically significant biomarkers or therapeutic targets for the diagnosis, prognosis and treatment of cardiovascular diseases. The pro-inflammatory properties of HIMF are of particular interest, as these contribute to metabolic complications which are closely associated with chronic low-grade inflammation. Similar to its function in lung tissue, HIMF regulates glucose and energetic metabolism in dose and cell-dependent manners. Increased HIMF expression results in metabolic disturbance, but basal levels of HIMF expression by a certain group of immune cells $\left(\mathrm{CD} 301 \mathrm{~b}^{+}\right.$mononuclear phagocytes) is required for the maintenance of metabolic homeostasis (Kumamoto et al., 2016).

This review summarizes the molecular characteristics of HIMF, including its molecular structure, tissue-specific distribution, transcription mechanisms and receptors. We primarily focus on the pluripotent physiological and pathological functions of HIMF, particularly in the pathogenesis of pulmonary, cardiovascular and metabolic disorders, alongside, the respective downstream signaling pathways. The potential clinical significance of HIMF is also discussed.

\section{MOLECULAR CHARACTERIZATIONS}

\section{Structure}

HIMF (RELM $\alpha$, also known as FIZZ1) is a $9.4 \mathrm{kDa}$ cysteine-rich secretory cytokine that belongs to the FIZZ/RELM family, which also includes RELM $\beta$ (FIZZ2) and RELM $\gamma$. RELM $\alpha$, and FIZZ1, as well as RELM $\beta$ and FIZZ2 were discovered independently by separate, unrelated labs as different functional proteins, but finally proved to be the same. The RELM proteins share a similar cysteine composition and other signature features with resistin (known as FIZZ3 in mice). The RELM family proteins typically range from 105 to 114 amino acids in length and are composed of three domains: an N-terminal sequence containing a secretory signal peptide, a variable middle portion, and a unique, highly conserved C-terminal signature sequence that contains 10 cysteine residues (Banerjee and Lazar, 2001). Members of the RELM family show strong interspecies and intraspecies homology, especially at this cysteine-rich C-terminus.

\section{Tissue-Specific Distribution}

The expression of HIMF, as with other members of the RELM family, is uneven across different types of tissue. For example, expression of HIMF (FIZZ1) is 10-fold higher in murine lung tissue compared with heart tissue or skeletal muscle (Holcomb et al., 2000). Alongside the lung and heart, HIMF is also expressed in the tongue, but its expression is highest in adipose tissue (Steppan et al., 2001). In addition, HIMF has also been found to mediate myeloid cell chemotaxis (Su et al., 2007). Meanwhile, RELM $\beta$ (FIZZ2) is exclusively expressed in the gastrointestinal tract, specifically in actively replicating crypt endothelium of the colon and small bowel (Hogan et al., 2006), similar to the findings by Holcomb. RELM $\gamma$ has been found to be expressed in hematopoietic tissues and lung in rodents (Gerstmayer et al., 2003), and FIZZ3 is expressed in white adipocytes throughout the body (Holcomb et al., 2000).

In addition to these initial findings, RELM proteins may also be expressed in other tissues under pathological conditions or during development. For example, HIMF and other RELM proteins are expressed in the murine liver during helminthinduced Th2-type immune responses (Pesce et al., 2009). HIMF is also expressed by macrophages, and increased HIMF levels can be used as a marker for alternatively activated (M2) macrophages in mice. RELM $\beta b$ and resistin are homologs of HIMF, and the expression patterns of RELM $\beta$ in the human lung are similar to those of HIMF in mice. Human resistin is also expressed in myeloid cells, especially macrophages, with a similar expression pattern to murine HIMF. Advances in protein quantification techniques are also revealing novel expression sites for RELM proteins, with hResistin expression recently being characterized across normal human tissues using a newly developed monoclonal antibody (Lin et al., 2020). hResistin was found to be principally localized in the cytoplasmic granules of macrophages, which are present in the interstitial space of the majority of human tissues. Therefore, the functions of HIMF in rodents may indicate potential roles of human RELM $\beta$ and resistin depending on the induction site and the cellular source of the protein.

\section{Transcription Mechanisms}

The mechanisms involved in the induction of HIMF transcription remain to be fully characterized, and may vary between physiological and pathological conditions. However, its expression is altered during development. For example, HIMF expression is upregulated in embryonic mouse lung tissue, and is involved in normal lung development. The transcription factor Ets-1, which is also expressed in the developing mouse lung, has been found to contribute to developmental expression of HIMF (Li et al., 2007). In this study, Ets- 1 was found to increase HIMF promoter luciferase activity in a heterogenous expression system, and chromatin immunoprecipitation (ChIP) assays revealed that Ets-1 bound to the HIMF promoter region in embryonic day (E) 20 lung tissues. Ets-1 is also known to participate in the transcriptional activation of vascular endothelial growth factor receptor-2 (Flk-1), in coordination with hypoxia-inducible factor $2 \alpha$ (HIF-2 $\alpha$ ), during vascular development and angiogenesis (Elvert et al., 2003). HIF-2 $\alpha$ expression is co-localized with HIMF in the developing airway epithelial cells and alveolar type II cells, suggesting that it may also induce HIMF expression during lung development (Wagner et al., 2004).

Furthermore, signal transducers and activators of transcription 6 (STAT6) and C/EBP have been suggested to mediate HIMF induction during the Th2 inflammatory response (Yamaji-Kegan et al., 2010). Cellular experiments demonstrated that HIMF promoter reporter gene constructs respond to Th2cytokines, IL-4, and IL-13 stimulation. In addition, the promoter region of the HIMF gene was found to contain functional binding sites for signal transducers and activators of transcription 6 (STAT6) and C/EBP. Point mutations in the STAT6 or the C/EBP 
sites led to the loss of cytokine responsiveness, indicating that Th2-related HIMF induction is orchestrated by the coordinated action of STAT6 and C/EBP. The involvement of STAT6 in the mediation of HIMF production has also been confirmed in a murine model of acute pulmonary inflammation, with HIMF found to be upregulated $6 \mathrm{~h}$ after antigen challenge. Notably, this effect was abolished by STAT6 gene ablation.

The potential role of hypoxia inducible factor-1 $\alpha$ (HIF$1 \alpha$ ) in HIMF induction, particularly in hypoxia-induced $\mathrm{PH}$, has also generated a great deal of attention. HIF- $1 \alpha$ is also activated during hypoxia, and serves a critical function in both hypoxic inflammation and Th2 immune activation in the lung. Previous experiments in HIF- $1 \alpha$ heterozygous null (HIF- $1 \alpha^{+/-}$) mice also found that HIMF induced HIF- $1 \alpha$ expression, and HIMF-induced PH was significantly diminished in HIF- $1 \alpha^{+/-}$ mice (Johns et al., 2016). In addition, recent work by our group revealed that HIMF increases HIF-1 $\alpha$ expression and cardiomyocyte hypertrophy, but HIF- $1 \alpha$ activation has no impact on HIMF expression (Kumar et al., 2018). Taken together, these results suggest that HIF- $1 \alpha$ is a critical downstream mediator of HIMF-induced $\mathrm{PH}$ and cardiac hypertrophy, rather than an upstream transcription factor controlling HIMF expression.

\section{Receptors}

Although a number of signaling pathways are activated by HIMF, the functional receptors for HIMF, resistin and other RELM proteins, remain unclear. Previous research has determined that HIMF induces the release of intracellular $\mathrm{Ca}^{2+}$ in pulmonary artery smooth muscle cells through the PLC-IP3 pathway (Fan et al., 2009). This suggests that HIMF may act as a ligand of $\mathrm{G}$ protein coupled receptors (GPCRs), the activation of which stimulates PLC-IP3 signaling. This previous study further revealed that the HIMF-induced $\mathrm{Ca}^{2+}$ response was attenuated by the tyrosine kinase inhibitor genistein. In addition, the pattern of $\mathrm{Ca}^{2+}$ release was altered by $\mathrm{G} \alpha_{\mathrm{q} / 11}$ knockdown, from sustained oscillatory $\mathrm{Ca}^{2+}$ transients with prolonged plateaus to a series of short $\mathrm{Ca}^{2+}$ transients. However, this effect was not elicited by $\mathrm{G} \alpha_{i}$ or $\mathrm{G} \alpha_{s}$ knockdown. These results led the authors to conclude that $\mathrm{G} \alpha_{\mathrm{q} / 11}$ protein-coupled receptors and a receptor tyrosine kinase are critical to HIMFinduced $\mathrm{Ca}^{2+}$ signaling. A more recent study from the same group demonstrated that Bruton's tyrosine kinase (BTK) is a binding partner for HIMF (Lin et al., 2019a), providing further evidence to suggest that HIMF functions via intracellular receptor tyrosine phosphorylation. However, the exact GPCRs involved remain unknown.

A recent study has reported that extracellular calciumsensing receptor (CaSR) acts as a receptor for intracellular HIMF (Zeng et al., 2017). Using a yeast 2-hybrid assay, the authors found that HIMF bound to the intracellular domain of CaSR, increasing the activity of the receptor. In turn, this mediated the hypoxia-induced proliferation of PSMCs, pulmonary vascular remodeling, and consequent pulmonary hypertension. However, CaSR appears to be a non-classical receptor for HIMF, mediating intracellular HIMF signaling only. Although the classical HIMF receptor remains to be identified, it is interesting that a synthesized, membrane-permeable peptide targeting the intracellular binding domain of CaSR for HIMF attenuated the development of hypoxia-induced $\mathrm{PH}$.

\section{PLUORIPOTENT EFFECTS AND ASSOCIATED SIGNALING PATHWAYS}

\section{Mitogenesis}

One of the most prominent functions of HIMF is its involvement in mitogenesis. Indeed, it was initially termed RELM $\alpha$, but was renamed after it was found to induce PSMC proliferation (Teng et al., 2003). Subsequent studies demonstrated that HIMF can also induce the proliferation of endothelial and fibroblast cells. The activation of the phosphatidylinositol 3-kinase (PI3K)/Akt pathway is critical during PSMC proliferation, with the inhibition of PI3K significantly suppressing Akt phosphorylation and PSMC proliferation. The PI3K/Akt pathway also contributes to the proliferation of HIMF-induced endothelial cells (ECs), by inducing the production of vascular endothelial growth factor (VEGF) through NF- $\mathrm{B}$ (Tong et al., 2006a; YamajiKegan et al., 2006). In addition, the mitogen-activated protein kinase (MAPK) pathway was also found to be involved in HIMF-induced EC proliferation, with the ERK inhibitor U0126 significantly inhibiting EC proliferation. A recent study by our group demonstrated that HIMF also stimulates cardiac fibroblast (CF) proliferation in a dose-dependent manner (Kumar et al., 2019). Here, IL-6 plays a key role, activating the MAPK and calcium/calmodulin-dependent protein kinase II (CaMKII)STAT3 pathways (Figure 1).

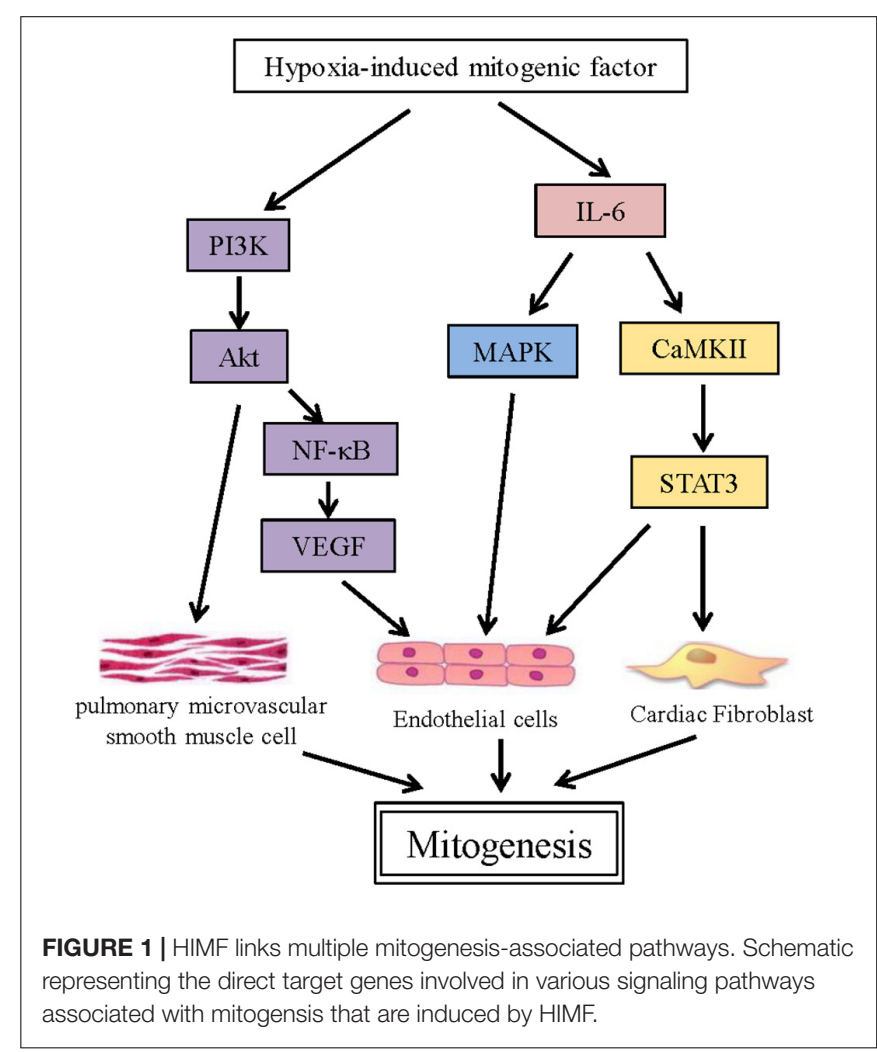




\section{Angiogenesis}

Angiogenesis involves a cascade of intricately regulated processes, including the dissolvement of the basement membrane and extracellular matrix, EC migration, and subsequent EC proliferation and vascular sprouting ( $\mathrm{Li}$ et al., 2013). The stimulation of angiogenic growth factors, including VEGFs and fibroblast growth factors (FGFs), plays an important role in this process ( $\mathrm{Li}$ et al., 2013). HIMF is also strongly associated with angiogenesis. Previous studies have shown that HIMF stimulates VEGF production in vascular ECs, and promotes the proliferation and migration of ECs and vascular sprouting (Tong et al., 2006c; Yamaji-Kegan et al., 2006). Furthermore, suppressing VEGF receptor-2 (VEGFR2) significantly inhibited HIMF-induced angiogenesis. HIMF has also been found to increase the production of Flk-1, which contributes to pulmonary angiogenesis (Tong et al., 2006b). Activation of the $\mathrm{PI}-3 \mathrm{~K} / \mathrm{Akt}-\mathrm{NF}-\kappa \mathrm{B}$ signaling pathway has been suggested to mediate the induction of VEGF and Flk-1 expression (Tong et al., 2006a,b). Notably, in addition to targeting endothelial cells directly, HIMF has also been shown to stimulate angiogenesis through the activation of myoblasts ( $\mathrm{Su}$ et al., 2017). HIMF increases IL-18 production through the PDK1/PI3K/Akt signaling pathway in myoblasts, which in turn promotes tube formation of the endothelial progenitor cells.

Angiogenesis is closely linked to inflammation. Proinflammatory cells, particularly macrophages, not only release large quantities of angiogenic factors under pathological conditions, but also indirectly promote angiogenesis through releasing inflammatory factors that recruit endothelial progenitor cells, which also promote vascular formation. HIMF has been found to recruit CD68-positive cells in mouse lungs and to stimulate the production of monocyte chemotactic protein-1 (MCP-1) and stromal cell-derived factor-1 (SDF-1), which are both angiogenesis-related inflammatory factors (Yamaji-Kegan et al., 2006). These effects were suppressed by treatment with a VEGFR2 inhibitor, providing further evidence to support a causal link between HIMF activity and VEGF-associated angiogenesis.

\section{Inflammation}

HIMF is a well-known marker of activated M2 anti-inflammatory macrophage. One of the most abundant neuropeptides in lung, calcitonin gene-related peptide (CGRP), has been found to attenuate lipopolysaccharide (LPS)-induced acute lung injury in rats. CGRP significantly reduced LPS-induced NLRP3 activation and increased the expression of HIMF, which was induced by IL-4 in macrophages (Duan et al., 2017). Similar results were reported in the liver of human cholestatic patients and bile duct-ligated mice. The activation of the NLRP3 inflammasome and increased numbers of M2 anti-inflammatory macrophages, as evidenced by increased HIMF expression, aggravated hepatic injury (Cai et al., 2020). Meanwhile, the inhibition of inflammation and induction of M2 macrophage polarization via the miR-223/TRAF6/NF- $\mathrm{B}$ axis alleviated viral myocarditis (Xue et al., 2020).

Previous studies have shown that HIMF induces the production and release of an array of pro-inflammatory factors, including IL-4 (Yamaji-Kegan et al., 2014), IL-6
(Johns et al., 2016), IL-18 (Su et al., 2017), TNF- $\alpha$ (Song et al., 2012), HMGB1 (Lin et al., 2019a,b), vascular adhesion molecule-1 VCAM-1 (Tong et al., 2006d), MCP-1 and SDF-1 (Yamaji-Kegan et al., 2010). A single injection of recombinant HIMF induced IL-4 production and lung injury in mice, while ablation of IL4 abolished the recruitment of macrophages to the lung and the pulmonary vascular inflammation caused by HIMF (YamajiKegan et al., 2014). In hypoxia-induced PH, HIMF induced the recruitment of macrophages and $\alpha$-SMA-production cells, and increased IL- 6 production via HIF- $1 \alpha$ activation (Johns et al., 2016). HIMF also upregulates VCAM-1 expression and mononuclear cell sequestration to the lung parenchyma in bacterial lipopolysaccharide (LPS)-induced acute lung injury, increasing its severity (Tong et al., 2006d). Furthermore, HIMF mediates EC-smooth muscle cell crosstalk, affecting HMGB1RAGE signaling (Lin et al., 2019a), and induces macrophagespecific HMGB1/RAGE expression. This, in turn, increases the apoptosis-resistant proliferation of human pulmonary artery smooth muscle cells during pulmonary vascular remodeling (Lin et al., 2019b). The induction of the pro-inflammatory factors MCP-1 and SDF-1 by HIMF also results in vascular remodeling in $\mathrm{PH}$. Meanwhile, in the heart, HIMF also stimulates IL-6 production in cardiomyocytes and cardiac fibroblasts via the activation of the MAPK and CaMKII-STAT3 pathways (Kumar et al., 2019).

Interestingly, HIMF appears to regulate T helper type 2 (Th2)induced inflammation in a different fashion. HIMF expression is induced by the Th2 cytokines IL- 4 and IL-13, and serves as a biomarker for the transformation of alternatively activated macrophages (AAMacs). The activation of AAMacs is the hallmark of several inflammatory conditions associated with parasite infection, allergy, diabetes and cancer (Nair et al., 2009). In a Schistosoma mansoni (Sm) eggs-induced mouse model of Th2 cytokine-dependent lung inflammation, HIMF suppressed Th2 cytokine-mediated pulmonary inflammation, and ablation of HIMF in mice exacerbated lung inflammation (Nair et al., 2009; Pesce et al., 2009). The effect of HIMF on the suppression of helminth-induced Th2-type immunity also occurs in other organs, such as the liver (Pesce et al., 2009). The underlying mechanism is related to the ability of HIMF inhibiting macrophage and $\mathrm{CD} 4^{+}$cells-mediated Th2 cytokine production in a Bruton's tyrosine kinase-dependent manner (Nair et al., 2009).

\section{Vasoconstriction}

In addition to the aforementioned processes, HIMF is also involved in vasoconstriction of the pulmonary artery. For example, intravenous injection of HIMF is known to increase pulmonary arterial pressure and pulmonary vascular resistance in mice. Surprisingly, the constrictive effect of HIMF is even more potent than either endothelin-1 or angiotensin II (Teng et al., 2003). This effect has been attributed to regulation of the $\mathrm{Ca}^{2+}$ signal by HIMF, with recombinant murine HIMF increasing the intracellular $\mathrm{Ca}^{2+}$ concentration in a sustained, oscillatory manner in human pulmonary artery smooth muscle cells (SMCs). Notably, the $\mathrm{Ca}^{2+}$ increase is related to $\mathrm{IP}_{3}$ mediated intracellular $\mathrm{Ca}^{2+}$ release, but not extracellular $\mathrm{Ca}^{2+}$ 
influx. Inhibition of $\mathrm{PLC}^{-\mathrm{IP}_{3}}$ and tyrosine kinase abolished HIMF-induced $\mathrm{Ca}^{2+}$ signaling, while knockdown of $\mathrm{G} \alpha_{\mathrm{q} / 11}$ expression and ryanodine pre-treatment altered the pattern of $\mathrm{Ca}^{2+}$ release (Fan et al., 2009). Taken together, this suggests that HIMF stimulates intracellular $\mathrm{Ca}^{2+}$ release in human pulmonary artery SMCs through the PLC signaling pathway, in an IP3and tyrosine phosphorylation-dependent manner. Furthermore, $\mathrm{G} \alpha_{\mathrm{q} / 11}$ protein-coupled receptors and ryanodine receptors are also involved in $\mathrm{Ca}^{2+}$ regulation.

However, whether HIMF exerts similar effects on systemic artery SMCs, and the potential roles of HIMF in regulation of blood pressure, remain to be fully elucidated and warrant further study.

\section{HIMF AND DISEASES}

\section{Pulmonary Diseases Pulmonary Hypertension (PH)}

The most well-studied disease associated with HIMF is hypoxia-induced $\mathrm{PH}$; a disease characterized by the progressive elevation of hypoxia-induced pulmonary vascular resistance, the development of right ventricular failure, and ultimately death. The elevation of vascular resistance is primarily attributed to pulmonary artery constriction and vascular remodeling in response to hypoxia. Vascular remodeling involves complicated pathological processes that include angiogenesis, muscularization, the thickening of small pulmonary vessels, inflammation, and fibrosis. HIMF is upregulated in the pulmonary vasculature, bronchial epithelial cells, and type II pneumocytes in hypoxia-induced PH (Teng et al., 2003). HIMF is also known to increase pulmonary vasoconstriction, and has a critical role in each pathological process of vascular remodeling. It enhances the angiogenic capacity of pulmonary myoblasts and the tubule formation of progenitor endothelial cells (Su et al., 2017). Furthermore, HIMF also induces the proliferation of pulmonary SMCs and fibroblast (FCs), as well as the differentiation of FCs, leading to muscularization, thickening of small pulmonary vessels, and lung fibrosis (Teng et al., 2003). In addition, HIMF induces lung inflammation, which promotes vascular remodeling and lung fibrosis (Liu et al., 2009; Angelini et al., 2013; Figure 2).

The human homolog of HIMF, RELM $\beta$, has been shown to be upregulated in the lung tissue of patients with sclerodermaassociated pulmonary hypertension (Angelini et al., 2009). RELM $\beta$ is primarily localized in the endothelium and vascular smooth muscle of remodeled vessels and in plexiform lesions, macrophages, $\mathrm{T}$ cells, and myofibroblast-like cells; similar to HIMF in hypoxia-induced $\mathrm{PH}$ in mice. Thus, clarifying the role of HIMF in the development of $\mathrm{PH}$ in mice may aid our understanding of the function of human RELM $\beta$ in the development of scleroderma-associated PH.

\section{Allergic Asthma}

HIMF was initially described as present in the bronchoalveolar lavage fluid in a murine allergic pulmonary inflammation model (Holcomb et al., 2000). HIMF production in antigen-challenged lungs has been suggested to be driven by either IL-4 or IL13, involving transcription factors STAT6 and C/EBP (Stutz et al., 2003; Yamaji-Kegan et al., 2010). Since the activation of STAT6 signaling has a critical role in allergic pulmonary inflammation, the STAT6-dependent induction of HIMF in the alveolar epithelium during pulmonary inflammation and fibrosis suggests that HIMF is involved in asthma pathogenesis. Supporting this, HIMF expression has since been found to be increased in OVA-induced inflammation (Fan et al., 2015). Furthermore, another previous study demonstrated that HIMF inhibited the nerve growth factor (NGF)-mediated survival of rat embryonic dorsal root ganglion (DRG) neurons, in addition to suppressing NGF-induced CGRP gene expression in adult rat DRG neurons (Holcomb et al., 2000). HIMF may modulate the function of neurons innervating the bronchial tree, and thus alter the local tissue response to allergic pulmonary inflammation. Further studies to clarify the role of HIMF in the inflammatory response in allergic asthma are clearly warranted.

\section{Cardiovascular Diseases}

\section{Cardiac Hypertrophy and Heart Failure}

A number of recent studies have demonstrated that HIMF has a critical role in the development of pressure overload-induced cardiac hypertrophy. For example, HIMF is upregulated in both a phenylephrine (PE)-stimulated cell model of cardiomyocyte hypertrophy, and a transverse aortic constriction (TAC)-induced mouse model of cardiac hypertrophy (Kumar et al., 2018). In this study, cardiomyocyte hypertrophy was induced by HIMF overexpression in cardiomyocytes, while knockdown of HIMF expression inhibited this process. In the TACinduced mouse model, ablation of the HIMF gene attenuated TAC-induced cardiac hypertrophy and fibrosis, and improved cardiac function. The activation of HIF- $1 \alpha$ and $\mathrm{Ca}^{2+}$-activated calcineurin (CaN)-nuclear factor of activated T cell (NFAT), as well as the MAPK pathway, contributed to the hypertrophic growth of cardiomyocytes. Interestingly, HIMF is not expressed by cardiac fibroblasts. However, the extracellular application of recombinant HIMF protein and conditioned medium from cultured cardiomyocytes overexpressing HIMF still induced fibroblast proliferation, differentiation, and migration. This indicates that HIMF produced in cardiomyocytes during the development of cardiac hypertrophy activates adjacent cardiac fibroblasts via paracrine signaling, inducing fibrosis (Kumar et al., 2019). Another previous study also demonstrated that serum levels of resistin, the human homolog of HIMF, are positively correlated with the severity of heart failure and the risk of adverse cardiac events in patients with heart failure (Zhang et al., 2011). While HIMF is not expressed in humans, the effects of HIMF on the development of cardiac hypertrophy and deterioration of cardiac function in mice may serve as a model for the function of resistin in the human heart under pathological conditions.

\section{Myocardial Infarction}

Recently, HIMF has been found to be involved in inhibiting apoptosis and increasing proliferation in cardiac fibroblasts, which result in cardiac fibrosis in a myocardial infarction (MI) mouse model. Furthermore, HIMF knockout conferred 


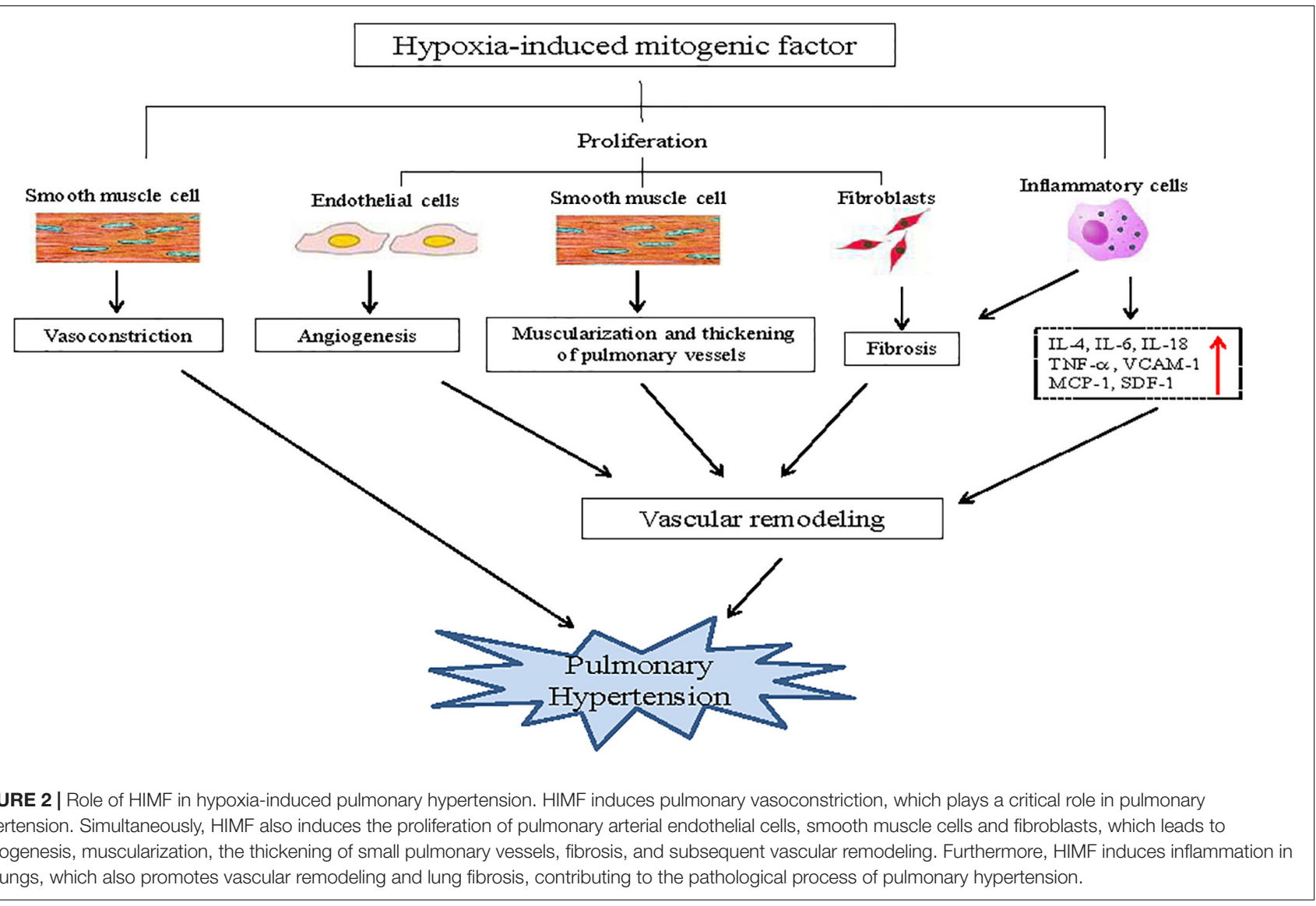

cardioprotective features on mice, with the suppression of cardiomyocyte apoptosis, fibroblast proliferation (Kim et al., 2021). These are contributed by adiponectin-enriched unsorted bone marrow cells (UBCs). Similarly, HIMF deficiency has also been found to reduce myocardial infarct size and improve cardiac function after MI (Li et al., 2021). HIMF overexpression also directly increased CHOP expression in BMDM and RAW264.7 cells, and regulated macrophage polarization (Li et al., 2021). Taken together, these studies suggest a complex role for HIMF during cardiac injury.

\section{Atherosclerosis}

HIMF increases EC and VSMC proliferation and, thus, angiogenesis. In addition, HIMF also induces vascular inflammation, which increases vascular permeability and cell adhesion to the endothelium. These functions suggest HIMF may also participate in the development and progression of atherosclerosis, although this has not been confirmed in vivo. However, resistin and RELM $\beta$, the two human homologs of HIMF, have been shown to play critical roles in atherosclerosis. Resistin is expressed in atherosclerotic plaque in patients (Jurin et al., 2018; Liberale et al., 2018), and its plasma level is linked to both coronary heart disease (Pourmoghaddas et al., 2020) and future cardiovascular-associated mortality (Park et al., 2017). At the cellular level, resistin upregulates the expression of inflammatory cytokines and adhesion molecules in human
ECs (Macdonald et al., 2017), induces SMC proliferation and migration, and promotes foam cell formation (Park et al., 2017). In vivo experiments on rabbits have also provided direct evidence that resistin aggravates atherosclerosis by stimulating monocytes, endothelial cells, and vascular smooth muscle cells, inducing vascular inflammation (Cho et al., 2011). Another previous study has also revealed the involvement of RELM $\beta$ in atherosclerotic progression (Kushiyama et al., 2013). RELM $\beta$ was abundantly expressed in foam cells within plaques from human samples. Furthermore, ablation of RELM $\beta$ significantly reduced lipid accumulation in the aortic root and wall in apolipoprotein E-deficient mice, and the inflammatory response of primary cultured peritoneal macrophages. This indicated that RELM $\beta$ contributes to atherosclerosis development via lipid accumulation and inflammatory facilitation. RELM $\beta$ has also previously been reported to contribute to the regulation of local immune responses in both gut (Ahmed et al., 2019) and bronchial epithelial cells (Fang et al., 2012). The discovery that it is also expressed in foam cells suggests that the tissue-specific distribution of RELM family members may not be particularly strict, and that these proteins can be expressed in unexpected tissues under pathological conditions.

\section{Metabolic Disorders}

The prolonged intraperitoneal administration of HIMF has been found to significantly increase insulin resistance in 
mice, but the mechanisms remain unknown (Al-Azzawi et al., 2007). Chronic low-grade inflammation is associated with metabolic complications associated during obesity, including insulin resistance. HIMF was readily detected in the serum at baseline, and its level was regulated by energy uptake, strongly suggesting that HIMF has a metabolic role. A previous study in a murine model of dextran sodium sulfate (DSS)-induced colitis and glucose disorder suggested that inflammation may be the mechanism underlying HIMF-induced dysregulation of glucose metabolism and energy balance (Munitz et al., 2009). HIMF serum levels were upregulated in DSS-induced colitis, and ablation of HIMF both decreased inflammation in situ and ameliorated DSS-induced colitis. The suppression of the inflammatory response leads to restoration of glucose tolerance, which is impaired in DSS-induced colitis, and protects the mice from hyperglycemia induced by glucose challenge. It support that HIMF contributes to glucose metabolism when it is induced during the setting of specific intestinal inflammatory conditions and the host is exposed to increased pro-inflammatory cytokines and high glucose intake.

While the lack of metabolic phenotype reported in HIMF knockout mice under steady-state conditions, these animals seem to have developed a compensatory mechanism to maintain normal metabolic homeostasis, as they maintain lower leptin concentrations in the sera despite their normal body weight and normal weight gain upon HFD feeding. Leptin plays an important role in regulating energy intake and expenditure, and has a pro-inflammatory role in colitis. The effect of HIMF to regulate leptin levels may also contribute to its overall pro-inflammatory role in vivo.

As HIMF serum levels increased in low-density lipoprotein receptor-deficient mice fed a high fat diet, HIMF exerted

TABLE 1 | Presentation of the studied diseases relating to HIMF.

\begin{tabular}{|c|c|c|c|}
\hline Author/study & Subjects & $\begin{array}{l}\text { Pathological } \\
\text { condition }\end{array}$ & Effects \\
\hline Teng et al. (2003) & $\begin{array}{l}\text { Rat pulmonary microvascular } \\
\text { smooth muscle cell }\end{array}$ & $\begin{array}{l}\text { Pulmonary } \\
\text { hypertension }\end{array}$ & $\begin{array}{l}\text { HIMF has angiogenic and vasoconstrictive properties by upregulating VEGF } \\
\text { production and promotes the proliferation and migration of PSMCs. }\end{array}$ \\
\hline Su et al. (2017) & $\begin{array}{l}\text { Murine myoblastic cell lines } \\
\text { (C2C12 and G7); Human } \\
\text { circulating EPCs; Male nude } \\
\text { mice }\end{array}$ & $\begin{array}{l}\text { Pulmonary } \\
\text { hypertension }\end{array}$ & $\begin{array}{l}\text { HIMF increased IL-18 production in myoblasts and promoted tube formation of } \\
\text { the endothelial progenitor cells. }\end{array}$ \\
\hline $\begin{array}{l}\text { Angelini et al. } \\
\text { (2013) }\end{array}$ & Adult C57BL/6 male mice & $\begin{array}{l}\text { Pulmonary } \\
\text { hypertension }\end{array}$ & $\begin{array}{l}\text { Pulmonary vascular remodeling in mice induced by chronic hypoxia or antigen } \\
\text { challenge is associated with marked increases in HIMF expression. }\end{array}$ \\
\hline Liu et al. (2009) & $\begin{array}{l}\text { Lung fibroblasts; FX knockout } \\
\text { C57BL/6 mice }\end{array}$ & $\begin{array}{l}\text { Pulmonary } \\
\text { hypertension }\end{array}$ & $\begin{array}{l}\text { Notch1 signaling in response to HIMF plays a significant role in myofibroblast } \\
\text { differentiation during lung fibrosis. }\end{array}$ \\
\hline $\begin{array}{l}\text { Holcomb et al. } \\
(2000)\end{array}$ & $\begin{array}{l}\text { Rat embryonic dorsal root } \\
\text { ganglion (DRG) neurons; BALB/c } \\
\text { female mice }\end{array}$ & Allergic asthma & $\begin{array}{l}\text { HIMF inhibited the nerve growth factor (NGF)-mediated survival of rat embryonic } \\
\text { dorsal root ganglion (DRG) neurons and NGF-induced CGRP gene expression } \\
\text { in adult rat DRG neurons. HIMF may modulate the function of neurons } \\
\text { innervating the bronchial tree, and thus alter the local tissue response to allergic } \\
\text { pulmonary inflammation. }\end{array}$ \\
\hline $\begin{array}{l}\text { Yamaji-Kegan et al. } \\
\text { (2010) }\end{array}$ & $\begin{array}{l}\text { IL-4 and STAT6 knockout } \\
\text { C57BL/6 male mice; Mouse } \\
\text { pulmonary microvascular } \\
\text { endothelial cells (PMVECs) }\end{array}$ & Lung inflammation & $\begin{array}{l}\text { IL-4 signaling may play a significant role in HIMF-induced lung inflammation and } \\
\text { vascular remodeling. }\end{array}$ \\
\hline Stutz et al. (2003) & $\begin{array}{l}\text { BALB/c female mice; BMnot cell } \\
\text { line }\end{array}$ & Allergic asthma & $\begin{array}{l}\text { STAT6 directly regulates IL-4- and IL-13-triggered induction of HIMF expression } \\
\text { at the transcriptional level by cooperation with C/EBP. }\end{array}$ \\
\hline Fan et al. (2015) & $\begin{array}{l}\text { RELMa knockout BALB/c male } \\
\text { Mice }\end{array}$ & Allergic asthma & $\begin{array}{l}\text { The expression of HIMF increased typically in OVA-induced pulmonary } \\
\text { inflammation and vascular remodeling. }\end{array}$ \\
\hline Kumar et al. (2018) & $\begin{array}{l}\text { HIMF knockout C57BL/6 male } \\
\text { mice; Neonatal ratventricular } \\
\text { myocytes }\end{array}$ & Cardiac hypertrophy & $\begin{array}{l}\text { HIMF has a critical role in the development of cardiac Hypertrophy via } \\
\text { calcium-dependent and HIF-1 } \alpha \text { Mechanisms. }\end{array}$ \\
\hline Kumar et al. (2019) & $\begin{array}{l}\text { HIMF knockout C57BL/6 male } \\
\text { mice; Neonatal rat ventricular } \\
\text { myocytes and Fibroblasts }\end{array}$ & Cardiac hypertrophy & $\begin{array}{l}\text { IL-6 plays a central role in HIMF-induced cardiac hypertrophy and fibrosis that is } \\
\text { mediated by activating the MAPK and CaMKII-STAT3 pathways. }\end{array}$ \\
\hline $\begin{array}{l}\text { Al-Azzawi et al. } \\
\text { (2007) }\end{array}$ & $\begin{array}{l}\text { C57BL/6J lean non-diabetic } \\
\text { female mice }\end{array}$ & Metabolic disorders & HIMF increases insulin resistance and reduces gallbladder optimal tension. \\
\hline Munitz et al. (2009) & $\begin{array}{l}\text { Retnla }{ }^{-/-} \text {male and female mice } \\
\text { (backcrossed to C57BL/6 or } \\
\text { BALB/c) }\end{array}$ & DSS-induced colitis & $\begin{array}{l}\text { HIMF deficiency reduced the colitis-induced systemic inflammatory response to } \\
\text { protect mouse from hyperglycemia induced by glucose injections. }\end{array}$ \\
\hline $\begin{array}{l}\text { Kumamoto et al. } \\
\text { (2016) }\end{array}$ & $\begin{array}{l}\text { Mgl2-DTR }\left(M g / 2+{ }^{-G F P}\right) \\
\text { C57BL/6 male mice }\end{array}$ & Metabolic disorders & $\begin{array}{l}\text { Reconstituting HIMF in CD301b+ MNP-depleted animals restored body weight } \\
\text { and normoglycemia. }\end{array}$ \\
\hline Lee et al. (2014) & $\begin{array}{l}\text { Ldlr } r^{-/}, \text {Ldlr } r^{-/-} \text {Retnla } \\
\text { Ldlr } r^{-/-} \text {Retnla-Tg C57BL/6J } \\
\text { male and female mice }\end{array}$ & $\begin{array}{l}\text { Hyperlipidemic and } \\
\text { atherosclerosis }\end{array}$ & $\begin{array}{l}\text { HIMF exerts a favorable cholesterol-lowering effect and protects against } \\
\text { atherosclerosis by enhancing cholesterol excretion in the form of bile acids in } \\
L d l r^{-/-} \text {mice. }\end{array}$ \\
\hline
\end{tabular}


a favorable cholesterol-lowering effect and conferred protection against atherosclerosis by increasing cholesterol excretion as bile acids (Lee et al., 2014). As demonstrated in a recent study (Kumamoto et al., 2016), a subset of

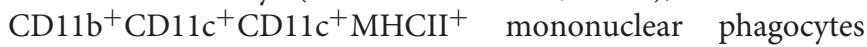
(MNP) characterized by the expression of CD301b (CD301b $\left.{ }^{+}\right)$, participate in the maintenance of normal glucose metabolism and energy balance through HIMF secretion. Deletion of CD301b ${ }^{+}$ MNPs in vivo leads to reduced HIMF expression, significant weight loss and increased insulin sensitivity. Reconstitution of HIMF expression in $\mathrm{CD}_{301 b^{+}}$MNP-ablated mice restores body weight and serum glucose level, and maintains the whole body metabolism. How HIMF secreted by CD301b ${ }^{+}$MNP controls feeding behavior and energy balance remains to be determined. These results suggest that the beneficial and detrimental roles of HIMF in inflammatory diseases are likely influenced by the type of immune stimulus, the duration of the stimulus exposure and the tissue type. However, the exact mechanisms underlying the involvement of HIMF in metabolic diseases remain to be clarified in full.

\section{CONCLUSION AND PERSPECTIVES}

As a secreted protein, HIMF exerts potent pro-inflammatory effects, is enriched at sites of inflammation, and its expression is correlated with markers of inflammatory disease. In recent years, the functional diversity of RELM proteins, such as HIMF, has generated increased attention. Mice are the main animal model used to manipulate the expression of these genes and to study their function. However, this also represents the biggest challenge faced when researching the role of RELM proteins and resistin in the pathogenesis of disease. The expression patterns of these proteins differ between humans and rodents; indeed, humans do not express HIMF at all. However, studies in mice do provide a solid starting point when investigating the function of RELM proteins in humans. HIMF has been suggested to play functional roles similar to those of human resistin, due to their similar expression patterns (Nair et al., 2006). If this is the case, the involvement of HIMF in the development of cardiovascular diseases and metabolic disorders in mice may be comparable to that of resistin in human disease. Using HIMF knockout mice could provide useful data that might indicate the role of resistin in these diseases. However, the situation may be different in the respiratory system. Here, HIMF appears to serve similar roles as human RELM beta: namely, inducing vascular remodeling and pulmonary fibrosis in pulmonary diseases, particularly in $\mathrm{PH}$. Therefore, HIMF data obtained in mice should be cautiously translated to human diseases, depending on the tissue localization and pathological context.

Early research has primarily focused on the involvement of HIMF in pulmonary vasoconstriction, vascular remodeling, pulmonary inflammation and angiogenesis, which are key pathophysiological processes that occur during the development of PH. HIMF has also been reported to be involved in the development of lung maturation, pulmonary fibrosis, acute lung injury and bronchial asthma. The expression and distribution of
HIMF could contribute to the initiation of diseases via multiple signaling pathways, which may cross-talk with each other in the development of pulmonary hypertension. A growing body of evidence also indicates a significant role for HIMF in lung disease. However, the underlying molecular mechanisms remain unclear in both pulmonary hypertension and other diseases. Additional studies are required to provide a more definitive account. Alternatively, whether HIMF is involved in other lung diseases, such as lung cancer, pneumonia, or chronic obstructive pulmonary disease (COPD) with emphysema, also warrants further investigation. A more thorough understanding of these mechanisms will be necessary in order to characterize HIMF, and its human homologs, as specific targets for the treatment of lung disease.

More recently, the study of RELM proteins in physiology and disease has expanded to include cardiovascular diseases. Clinical investigations and experimental studies have identified a positive correlation between circulating levels of resistin and the risk of MI. In mice, HIMF deficiency is known to facilitate M2 macrophage transformation and to increase collagen formation and fibrin deposition in the infarct region, preventing the myocardial wall from expansion and rupture. This helps preserve contractility after acute MI (Li et al., 2021). However, HIMF also induces cardiac fibroblast proliferation, migration and differentiation during cardiac hypertrophy and heart failure (Kumar et al., 2019). Additionally, HIMF may serve pathogenic functions in hypercholesterolemia (Lee et al., 2014) and immunemediated liver injury (Pai and Njoku, 2021). Thus, future studies on the pathogenic functions of HIMF should be extended to other pathological conditions. The main details of the studies focusing on the role of HIMF in diseases are demonstrated in Table $\mathbf{1 .}$

At present, the receptors for resistin and RELM proteins are not known. A recent study revealed a non-classical receptor for HIMF, CaSR, which mediates intracellular but not extracellular HIMF signal (Zeng et al., 2017). A synthesized membranepermeable peptide designed to flank the binding domain of CaSR for HIMF significantly attenuated hypoxia-induced $\mathrm{PH}$ progression. The therapeutic effect of this peptide on $\mathrm{PH}$ is quite inspiring, and there may be potential for its use to be extended to other HIMF-associated diseases. However, future studies focused on discovering the receptors and associated signaling pathways of RELM proteins will be required to fully understand its translational medical role in human diseases.

\section{AUTHOR CONTRIBUTIONS}

ML and WL prepared the figures. WL wrote the manuscript. Both authors contributed to the article and approved the submitted version.

\section{FUNDING}

The present study was supported by the National Science Foundation of China (82070340 to WL), the Guangdong Basic 
and Applied Basic Research Foundation (2020A1515010259 to WL), the Medical Scientific Research Foundation of Guangdong Province of China (A2016263 to WL), the SZU Top Ranking Project ( 86000000210 to WL), the Basic Research Foundation of Shenzhen (20200812105703001 to WL), and the SZU Medical Young Scientists Program (71201-000001 to WL).

\section{REFERENCES}

Ahmed, N., Heitlinger, E., Affinass, N., Kuhl, A. A., Xenophontos, N., Jarquin, V. H., et al. (2019). A Novel Non-invasive Method to Detect RELM Beta Transcript in Gut Barrier Related Changes During a Gastrointestinal Nematode Infection. Front. Immunol. 10:445. doi: 10.3389/fimmu.2019.00 445

Al-Azzawi, H. H., Mathur, A., Lu, D., Swartz-Basile, D. A., Nakeeb, A., and Pitt, H. A. (2007). Resistin-like molecule alpha reduces gallbladder optimal tension. J. Gastrointest. Surg. 11, 95-100. doi: 10.1007/s11605-006-0039-1

Angelini, D. J., Su, Q., Yamaji-Kegan, K., Fan, C., Skinner, J. T., Poloczek, A., et al. (2013). Hypoxia-induced mitogenic factor (HIMF/FIZZ1/RELMalpha) in chronic hypoxia- and antigen-mediated pulmonary vascular remodeling. Respir. Res. 14:1. doi: 10.1186/1465-9921-14-1

Angelini, D. J., Su, Q., Yamaji-Kegan, K., Fan, C., Teng, X., Hassoun, P. M., et al. (2009). Resistin-like molecule-beta in scleroderma-associated pulmonary hypertension. Am. J. Respir. Cell Mol. Biol. 41, 553-561. doi: 10.1165/rcmb. 2008-0271OC

Banerjee, R. R., and Lazar, M. A. (2001). Dimerization of resistin and resistin-like molecules is determined by a single cysteine. J. Biol. Chem. 276, 25970-25973. doi: 10.1074/jbc.M103109200

Cai, S. Y., Ge, M., Mennone, A., Hoque, R., Ouyang, X., and Boyer, J. L. (2020). Inflammasome Is Activated in the Liver of Cholestatic Patients and Aggravates Hepatic Injury in Bile Duct-Ligated Mouse. Cell Mol. Gastroenterol. Hepatol. 9, 679-688. doi: 10.1016/j.jcmgh.2019.12.008

Cho, Y., Lee, S. E., Lee, H. C., Hur, J., Lee, S., Youn, S. W., et al. (2011). Adipokine resistin is a key player to modulate monocytes, endothelial cells, and smooth muscle cells, leading to progression of atherosclerosis in rabbit carotid artery. J. Am. Coll. Cardiol. 57, 99-109. doi: 10.1016/j.jacc.2010.07.035

Duan, J. X., Zhou, Y., Zhou, A. Y., Guan, X. X., Liu, T., Yang, H. H., et al. (2017). Calcitonin gene-related peptide exerts anti-inflammatory property through regulating murine macrophages polarization in vitro. Mol. Immunol. 91, 105113. doi: $10.1016 /$ j.molimm.2017.08.020

Elvert, G., Kappel, A., Heidenreich, R., Englmeier, U., Lanz, S., Acker, T., et al. (2003). Cooperative interaction of hypoxia-inducible factor-2alpha (HIF2alpha) and Ets-1 in the transcriptional activation of vascular endothelial growth factor receptor-2 (Flk-1). J. Biol. Chem. 278, 7520-7530. doi: 10.1074/ jbc.M211298200

Fan, C., Meuchel, L. W., Su, Q., Angelini, D. J., Zhang, A., Cheadle, C., et al. (2015). Resistin-Like Molecule alpha in Allergen-Induced Pulmonary Vascular Remodeling. Am. J. Respir. Cell Mol. Biol. 53, 303-313. doi: 10.1165/rcmb.2014$0322 \mathrm{OC}$

Fan, C., Su, Q., Li, Y., Liang, L., Angelini, D. J., Guggino, W. B., et al. (2009). Hypoxia-induced mitogenic factor/FIZZ1 induces intracellular calcium release through the PLC-IP(3) pathway. Am. J. Physiol. Lung Cell Mol. Physiol. 297, L263-L270. doi: 10.1152/ajplung.90416.2008

Fang, C., Meng, Q., Wu, H., Eid, G., Zhang, G., Zhang, X., et al. (2012). Resistinlike molecule-beta is a human airway remodelling mediator. Eur. Respir. J. 39, 458-466. doi: 10.1183/09031936.00107811

Gerstmayer, B., Kusters, D., Gebel, S., Muller, T., Van Miert, E., Hofmann, K., et al. (2003). Identification of RELMgamma, a novel resistin-like molecule with a distinct expression pattern. Genomics 81, 588-595. doi: 10.1016/s0888-7543(03) 00070-3

Hogan, S. P., Seidu, L., Blanchard, C., Groschwitz, K., Mishra, A., Karow, M. L., et al. (2006). Resistin-like molecule beta regulates innate colonic function: barrier integrity and inflammation susceptibility. J. Allergy Clin. Immunol. 118, 257-268. doi: 10.1016/j.jaci.2006.04.039

Holcomb, I. N., Kabakoff, R. C., Chan, B., Baker, T. W., Gurney, A., Henzel, W., et al. (2000). FIZZ1, a novel cysteine-rich secreted protein associated with

\section{ACKNOWLEDGMENTS}

We appreciate Dr. Jessica Tamanini (Shenzhen University Health Science Center, China and ETediting, United Kingdom) for language editing and critical comments of the article before submission.

pulmonary inflammation, defines a new gene family. EMBO J. 19, 4046-4055. doi: 10.1093/emboj/19.15.4046

Johns, R. A., Takimoto, E., Meuchel, L. W., Elsaigh, E., Zhang, A., Heller, N. M., et al. (2016). Hypoxia-Inducible Factor lalpha Is a Critical Downstream Mediator for Hypoxia-Induced Mitogenic Factor (FIZZ1/RELMalpha)-Induced Pulmonary Hypertension. Arterioscler. Thromb. Vasc. Biol. 36, 134-144. doi: 10.1161/ATVBAHA.115.306710

Jurin, I., Paic, F., Bulimbasic, S., Rudez, I., Derek, L., Jurin, H., et al. (2018). Association between Circulatory and Plaque Resistin Levels with Carotid Plaque Instability and Ischemic Stroke Events. Heart. Surg. Forum 21, E448E463. doi: 10.1532/hsf.2071

Kim, Y. S., Cho, H. H., Cho, D. I, Jeong, H. Y., Lim, S. Y., Jun, J. H., et al. (2021). The adipokine Retnla deficiency increases responsiveness to cardiac repair through adiponectin-rich bone marrow cells. Cell Death Dis. 12:307. doi: 10.1038/s41419-021-03593-Z

Kumamoto, Y., Camporez, J. P. G., Jurczak, M. J., Shanabrough, M., Horvath, T., Shulman, G. I., et al. (2016). CD301b(+) Mononuclear Phagocytes Maintain Positive Energy Balance through Secretion of Resistin-like Molecule Alpha. Immunity 45, 583-596. doi: 10.1016/j.immuni.2016.08.002

Kumar, S., Wang, G., Liu, W., Ding, W., Dong, M., Zheng, N., et al. (2018). Hypoxia-Induced Mitogenic Factor Promotes Cardiac Hypertrophy via Calcium-Dependent and Hypoxia-Inducible Factor-1alpha Mechanisms. Hypertension 72, 331-342. doi: 10.1161/HYPERTENSIONAHA.118.10845

Kumar, S., Wang, G., Zheng, N., Cheng, W., Ouyang, K., Lin, H., et al. (2019). HIMF (Hypoxia-Induced Mitogenic Factor)-IL (Interleukin)6 Signaling Mediates Cardiomyocyte-Fibroblast Crosstalk to Promote Cardiac Hypertrophy and Fibrosis. Hypertension 73, 1058-1070. doi: 10.1161/HYPERTENSIONAHA.118.12267

Kushiyama, A., Sakoda, H., Oue, N., Okubo, M., Nakatsu, Y., Ono, H., et al. (2013). Resistin-like molecule beta is abundantly expressed in foam cells and is involved in atherosclerosis development. Arterioscler. Thromb. Vasc. Biol. 33, 1986-1993. doi: 10.1161/ATVBAHA.113.301546

Lee, M. R., Lim, C. J., Lee, Y. H., Park, J. G., Sonn, S. K., Lee, M. N., et al. (2014). The adipokine Retnla modulates cholesterol homeostasis in hyperlipidemic mice. Nat. Commun. 5:4410. doi: 10.1038/ncomms5410

Li, B., Lager, J., Wang, D., and Li, D. (2007). Ets-1 participates in and facilitates developmental expression of hypoxia-induced mitogenic factor in mouse lung. Front. Biosci. 12, 2269-2278. doi: 10.2741/2229

Li, X., Yang, Y., Fang, J., and Zhang, H. (2013). FIZZ1 could enhance the angiogenic ability of rat aortic endothelial cells. Int. J. Clin. Exp. Pathol. 6, 1847-1853.

Li, Y., Dong, M., Wang, Q., Kumar, S., Zhang, R., Cheng, W., et al. (2021). HIMF deletion ameliorates acute myocardial ischemic injury by promoting macrophage transformation to reparative subtype. Basic Res. Cardiol. 116:30. doi: 10.1007/s00395-021-00867-7

Liberale, L., Bertolotto, M., Carbone, F., Contini, P., Wust, P., Spinella, G., et al. (2018). Resistin exerts a beneficial role in atherosclerotic plaque inflammation by inhibiting neutrophil migration. Int. J. Cardiol. 272, 13-19. doi: 10.1016/j. ijcard.2018.07.112

Lin, Q., Fan, C., Gomez-Arroyo, J., Van Raemdonck, K., Meuchel, L. W., Skinner, J. T., et al. (2019a). HIMF (Hypoxia-Induced Mitogenic Factor) Signaling Mediates the HMGB1 (High Mobility Group Box 1)-Dependent Endothelial and Smooth Muscle Cell Crosstalk in Pulmonary Hypertension. Arterioscler. Thromb. Vasc. Biol. 39, 2505-2519. doi: 10.1161/ATVBAHA.119.312907

Lin, Q., Fan, C., Skinner, J. T., Hunter, E. N., Macdonald, A. A., Illei, P. B., et al. (2019b). RELMalpha Licenses Macrophages for Damage-Associated Molecular Pattern Activation to Instigate Pulmonary Vascular Remodeling. J. Immunol. 203, 2862-2871. doi: 10.4049/jimmunol.1900535

Lin, Q., Price, S. A., Skinner, J. T., Hu, B., Fan, C., Yamaji-Kegan, K., et al. (2020). Systemic evaluation and localization of resistin expression in normal human 
tissues by a newly developed monoclonal antibody. PLoS One 15:e0235546. doi: 10.1371/journal.pone.0235546

Liu, T., Hu, B., Choi, Y. Y., Chung, M., Ullenbruch, M., Yu, H., et al. (2009). Notch1 signaling in FIZZ1 induction of myofibroblast differentiation. Am. J. Pathol. 174, 1745-1755. doi: 10.2353/ajpath.2009.080618

Macdonald, S. P. J., Bosio, E., Neil, C., Arendts, G., Burrows, S., Smart, L., et al. (2017). Resistin and NGAL are associated with inflammatory response, endothelial activation and clinical outcomes in sepsis. Inflamm. Res. 66, 611619. doi: 10.1007/s00011-017-1043-5

Munitz, A., Seidu, L., Cole, E. T., Ahrens, R., Hogan, S. P., and Rothenberg, M. E. (2009). Resistin-like molecule alpha decreases glucose tolerance during intestinal inflammation. J. Immunol. 182, 2357-2363. doi: 10.4049/jimmunol. 0803130

Nair, M. G., Du, Y., Perrigoue, J. G., Zaph, C., Taylor, J. J., Goldschmidt, M., et al. (2009). Alternatively activated macrophage-derived RELM-\{alpha\} is a negative regulator of type 2 inflammation in the lung. J. Exp. Med. 206, 937-952. doi: $10.1084 /$ jem.20082048

Nair, M. G., Guild, K. J., and Artis, D. (2006). Novel effector molecules in type 2 inflammation: lessons drawn from helminth infection and allergy. J. Immunol. 177, 1393-1399. doi: 10.4049/jimmunol.177.3.1393

Pai, S., and Njoku, D. B. (2021). The Role of Hypoxia-Induced Mitogenic Factor in Organ-Specific Inflammation in the Lung and Liver: Key Concepts and Gaps in Knowledge Regarding Molecular Mechanisms of Acute or Immune-Mediated Liver Injury. Int. J. Mol. Sci. 22:2717. doi: 10.3390/ijms22052717

Park, H. K., Kwak, M. K., Kim, H. J., and Ahima, R. S. (2017). Linking resistin, inflammation, and cardiometabolic diseases. Korean J. Intern. Med. 32, 239247. doi: 10.3904/kjim.2016.229

Pesce, J. T., Ramalingam, T. R., Wilson, M. S., Mentink-Kane, M. M., Thompson, R. W., Cheever, A. W., et al. (2009). Retnla (relmalpha/fizz1) suppresses helminth-induced Th2-type immunity. PLoS Pathog. 5:e1000393. doi: 10.1371/ journal.ppat.1000393

Pourmoghaddas, A., Elahifar, A., Darabi, F., Movahedian, A., Amirpour, A., and Sarrafzadegan, N. (2020). Resistin and prooxidant-antioxidant balance: markers to discriminate acute coronary syndrome from stable angina. ARYA Atheroscler. 16, 46-54. doi: 10.22122/arya.v16i2.1944

Song, L., Xu, J., Qu, J., Sai, Y., Chen, C., Yu, L., et al. (2012). A therapeutic role for mesenchymal stem cells in acute lung injury independent of hypoxia-induced mitogenic factor. J. Cell Mol. Med. 16, 376-385. doi: 10.1111/j.1582-4934.2011. 01326.x

Steppan, C. M., Brown, E. J., Wright, C. M., Bhat, S., Banerjee, R. R., Dai, C. Y., et al. (2001). A family of tissue-specific resistin-like molecules. Proc. Natl. Acad. Sci. U. S. A. 98, 502-506. doi: 10.1073/pnas.98.2.502

Stutz, A. M., Pickart, L. A., Trifilieff, A., Baumruker, T., Prieschl-Strassmayr, E., and Woisetschlager, M. (2003). The Th2 cell cytokines IL-4 and IL-13 regulate found in inflammatory zone $1 /$ resistin-like molecule alpha gene expression by a STAT6 and CCAAT/enhancer-binding protein-dependent mechanism. J. Immunol. 170, 1789-1796. doi: 10.4049/jimmunol.170.4.1789

Su, C. M., Wang, I. C., Liu, S. C., Sun, Y., Jin, L., Wang, S. W., et al. (2017). Hypoxia induced mitogenic factor (HIMF) triggers angiogenesis by increasing interleukin-18 production in myoblasts. Sci. Rep. 7:7393. doi: 10.1038/s41598017-07952-9

Su, Q., Zhou, Y., and Johns, R. A. (2007). Bruton's tyrosine kinase (BTK) is a binding partner for hypoxia induced mitogenic factor (HIMF/FIZZ1) and mediates myeloid cell chemotaxis. FASEB J. 21, 1376-1382. doi: 10.1096/fj.06$6527 \mathrm{com}$

Teng, X., Li, D., Champion, H. C., and Johns, R. A. (2003). FIZZ1/RELMalpha, a novel hypoxia-induced mitogenic factor in lung with vasoconstrictive and angiogenic properties. Circ. Res. 92, 1065-1067. doi: 10.1161/01.RES. 0000073999.07698 .33
Tong, Q., Zheng, L., Kang, Q., Dodd, O. J., Langer, J., Li, B., et al. (2006d). Upregulation of hypoxia-induced mitogenic factor in bacterial lipopolysaccharide-induced acute lung injury. FEBS Lett. 580, 2207-2215. doi: 10.1016/j.febslet.2006.03.027

Tong, Q., Zheng, L., Li, B., Wang, D., Huang, C., Matuschak, G. M., et al. (2006c). Hypoxia-induced mitogenic factor enhances angiogenesis by promoting proliferation and migration of endothelial cells. Exp. Cell. Res. 312, 3559-3569. doi: 10.1016/j.yexcr.2006.07.024

Tong, Q., Zheng, L., Lin, L., Li, B., Wang, D., Huang, C., et al. (2006a). VEGF is upregulated by hypoxia-induced mitogenic factor via the PI-3K/AktNF-kappaB signaling pathway. Respir. Res. 7:37. doi: 10.1186/1465-99217-37

Tong, Q., Zheng, L., Lin, L., Li, B., Wang, D., Huang, C., et al. (2006b). Participation of the PI-3K/Akt-NF-kappa B signaling pathways in hypoxiainduced mitogenic factor-stimulated Flk-1 expression in endothelial cells. Respir. Res. 7:101. doi: 10.1186/1465-9921-7-101

Wagner, K. F., Hellberg, A. K., Balenger, S., Depping, R., Dodd, O. J., Johns, R. A., et al. (2004). Hypoxia-induced mitogenic factor has antiapoptotic action and is upregulated in the developing lung: coexpression with hypoxia-inducible factor-2alpha. Am. J. Respir. Cell Mol. Biol. 31, 276-282. doi: 10.1165/rcmb. 2003-03190C

Xue, Y. L., Zhang, S. X., Zheng, C. F., Li, Y. F., Zhang, L. H., Su, Q. Y., et al. (2020). Long non-coding RNA MEG3 inhibits M2 macrophage polarization by activating TRAF6 via microRNA-223 down-regulation in viral myocarditis. J. Cell Mol. Med. 24, 12341-12354. doi: 10.1111/jcmm.15720

Yamaji-Kegan, K., Su, Q., Angelini, D. J., Champion, H. C., and Johns, R. A. (2006). Hypoxia-induced mitogenic factor has proangiogenic and proinflammatory effects in the lung via VEGF and VEGF receptor-2. Am. J. Physiol. Lung Cell Mol. Physiol. 291, L1159-L1168. doi: 10.1152/ajplung.00168.2006

Yamaji-Kegan, K., Su, Q., Angelini, D. J., Myers, A. C., Cheadle, C., and Johns, R. A. (2010). Hypoxia-induced mitogenic factor (HIMF/FIZZ1/RELMalpha) increases lung inflammation and activates pulmonary microvascular endothelial cells via an IL-4-dependent mechanism. J. Immunol. 185, 5539-5548. doi: 10.4049/jimmunol.0904021

Yamaji-Kegan, K., Takimoto, E., Zhang, A., Weiner, N. C., Meuchel, L. W., Berger, A. E., et al. (2014). Hypoxia-induced mitogenic factor (FIZZ1/RELMalpha) induces endothelial cell apoptosis and subsequent interleukin-4-dependent pulmonary hypertension. Am. J. Physiol. Lung Cell Mol. Physiol. 306, L1090L1103. doi: 10.1152/ajplung.00279.2013

Zeng, X., Zhu, L., Xiao, R., Liu, B., Sun, M., Liu, F., et al. (2017). Hypoxia-Induced Mitogenic Factor Acts as a Non-classical Ligand of Calcium-Sensing Receptor, Therapeutically Exploitable for Intermittent Hypoxia-Induced Pulmonary Hypertension. Hypertension 69, 844-854. doi: 10.1161/HYPERTENSIONAHA. 116.08743

Zhang, M. H., Na, B., Schiller, N. B., and Whooley, M. A. (2011). Association of resistin with heart failure and mortality in patients with stable coronary heart disease: data from the heart and soul study. J. Card. Fail. 17, 24-30. doi: 10.1016/j.cardfail.2010.08.007

Conflict of Interest: The authors declare that the research was conducted in the absence of any commercial or financial relationships that could be construed as a potential conflict of interest.

Copyright (c) $2021 \mathrm{Lv}$ and Liu. This is an open-access article distributed under the terms of the Creative Commons Attribution License (CC BY). The use, distribution or reproduction in other forums is permitted, provided the original author(s) and the copyright owner(s) are credited and that the original publication in this journal is cited, in accordance with accepted academic practice. No use, distribution or reproduction is permitted which does not comply with these terms. 